\title{
Esophageal adenocarcinoma with leukemoid reaction: a case report
}

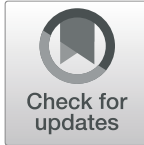

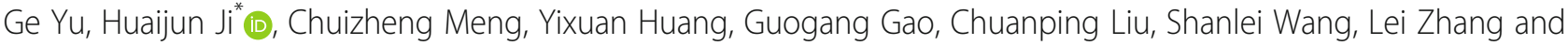 \\ Jin Ju*
}

\begin{abstract}
Background: Leukemoid reaction (LR) is defined as a reactive leucocytosis with WBC counts exceeding 50,000/ $\mathrm{mm}^{3}$, and a significant increase in early neutrophil precursors. LR may be a paraneoplastic manifestation of various malignant tumors. Tumor-related LR is a kind of neoplastic syndrome, unrelated to an infection or other diseases.

Case presentation: A 74-year-old male visited a local doctor with a 20-day history of progressive dysphagia. The complete blood count revealed leucocytosis. Bone marrow aspirates and a biopsy confirmed LR and excluded chronic myelogenous leukemia. Following radical esophagectomy for an adenocarcinoma the WBC counts successively decreased to $10,450 / \mathrm{mm}^{3}$ and $8670 / \mathrm{mm}^{3}$ within 1 week and 1 month, respectively.

Conclusion: We report a rare case of esophageal adenocarcinoma complicated with excessive leucocytosis caused by paraneoplastic LR; we also present a review of literature and an investigation of the clinical features. To our knowledge, this is the first report of LR associated with esophageal adenocarcinoma.
\end{abstract}

Keywords: Leukemoid reaction (LR), Hyperleukocytosis, Esophageal adenocarcinoma, Esophagectomy

\section{Background}

A leukemoid reaction (LR) is defined as white blood cell (WBC) count exceeding $50,000 / \mathrm{mm}^{3}$, owing to extramedullary causes [1]. LR is most often caused by serious infection [2], but can also be a paraneoplastic manifestation of several cancers, including lung, gastrointestinal, genitourinary, ovarian, and head and neck cancers, and hepatocellular carcinoma [3]. Hughes and Charles first reported a case of LR resulting from a carcinoma of the suprarenal gland in 1952 [4]. Since then, great efforts have been made to elucidate the mechanism of LR. Paraneoplastic syndromes, such as tumour fever and LR, have been described in literature $[5,6]$. However, studies on the incidence and course of LR in solid tumours are scarce [7]. One study reported that the occurrence of LR in solid tumours is approximately $1-4 \%$ [8]. However, owing to few accounts in medical literature, the causes of this occurrence are not well understood. Here, we report a rare case of esophageal adenocarcinoma complicated with

\footnotetext{
* Correspondence: sdhzcxjhj@sina.com; whxwkjj@sina.com

Department of Thoracic Surgery, Weihai Municipal Hospital, 70 Heping Road, Weihai 264200, Shandong, China
}

hyperleukocytosis caused by a paraneoplastic leukemoid reaction (PLR).

\section{Case presentation}

A 74-year-old male visited a local doctor in May 2018 due to a 20-day history of progressive dysphagia. He had no other symptoms suggestive of esophageal cancer. Electronic gastroscopy showed an elevated tumour in the lower segment of his esophagus, which revealed an esophageal adenocarcinoma on biopsy. The complete blood count revealed a total leucocyte count of 24,870 / $\mathrm{mm}^{3}$ and the peripheral blood smear showed differential counts of 89,5 , and $4 \%$ for neutrophils, lymphocytes, and monocytes $4 \%$, respectively. Since he had no fever, the treating physician did not suspect an inflammatory reaction or abscess, but considered this to be a manifestation of a secondary haematologica disorder. Chest computed tomography (CT) showed thickening of the wall of the esophagus, pulmonary inflammation, and mild bronchiectasis. Aspirates and a biopsy from the bone marrow (Fig. 1a) revealed granulocytosis that had proliferated actively, abundant droplet bodies in the cytoplasm, and an increased number of megakaryocytes. The positivity rate of alkaline phosphatase expression 
A

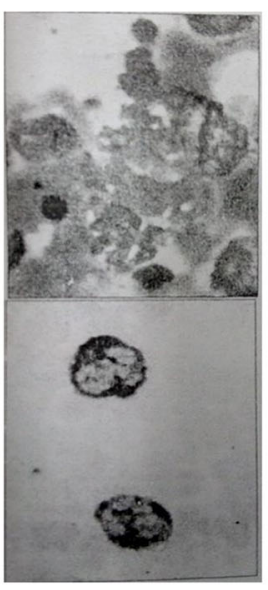

B
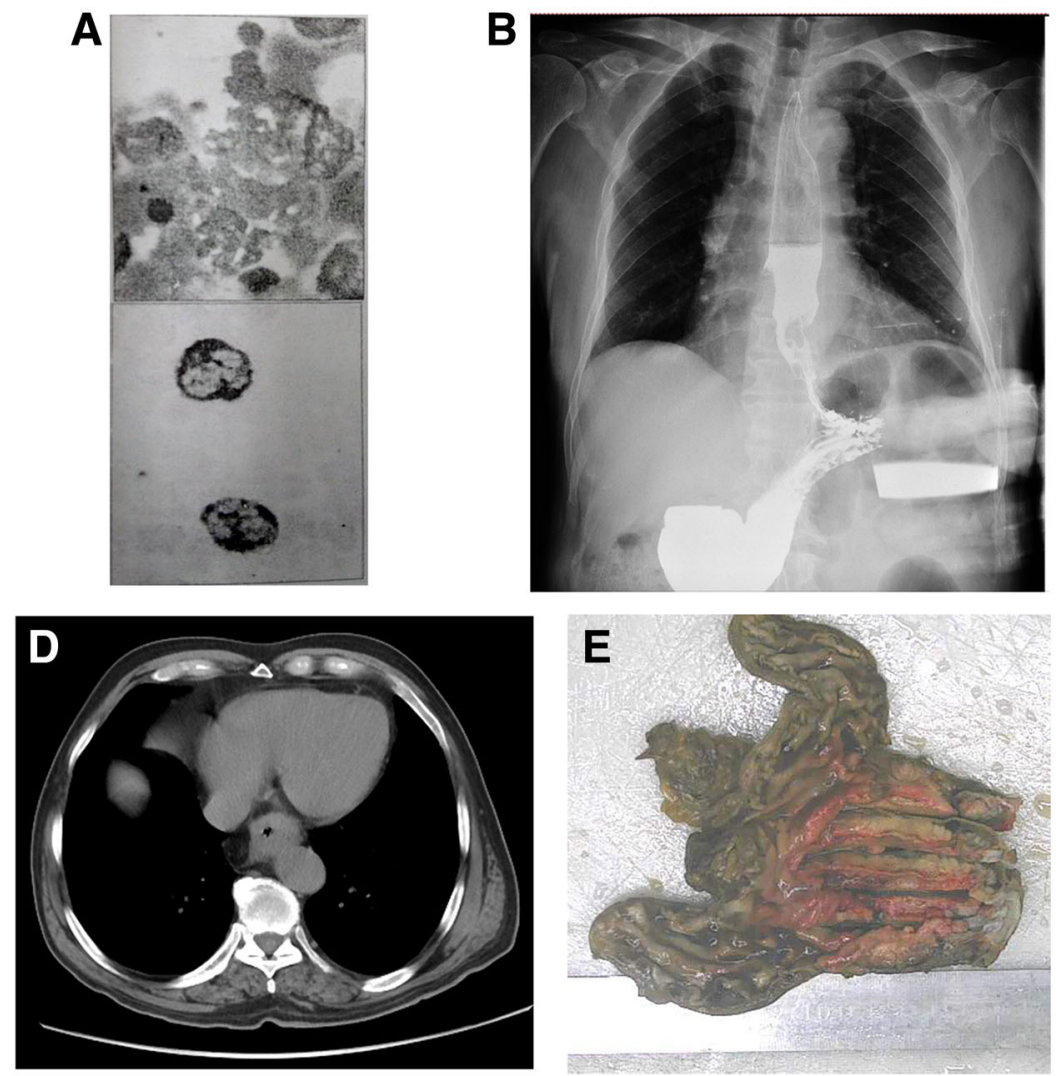

E

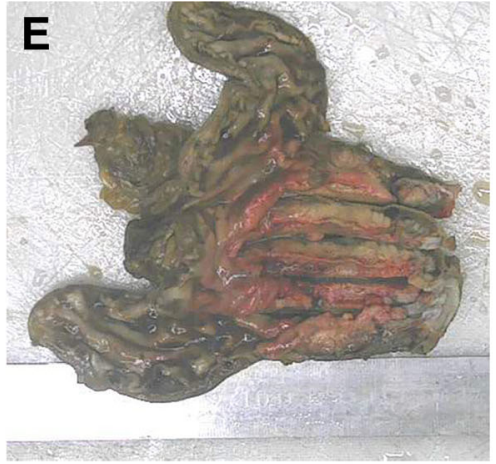

C
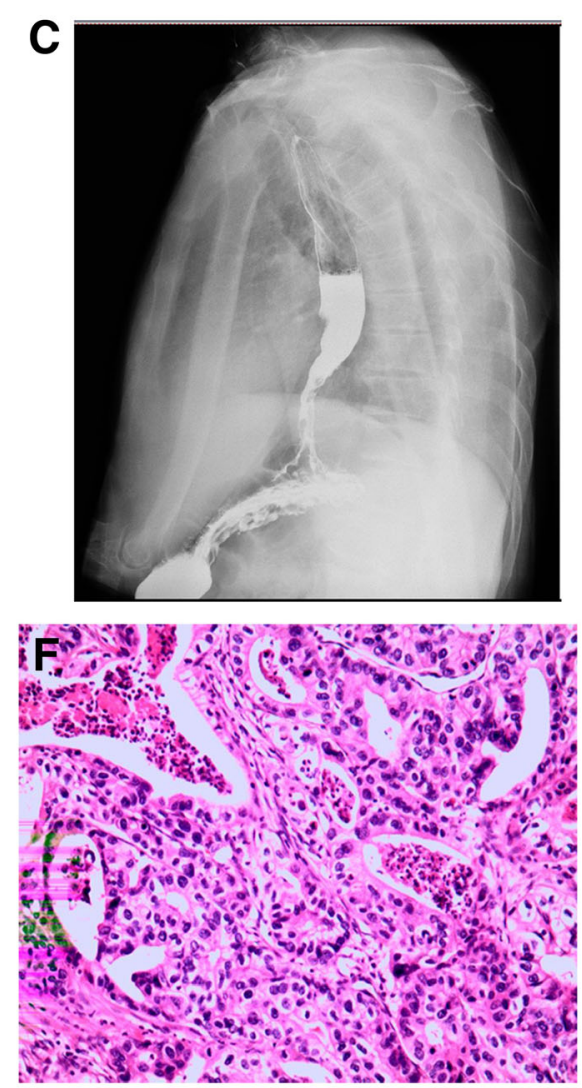

Fig. 1 Test results of a patient with paraneoplastic leukemoid reaction associated with esophageal adenocarcinoma. a Bone marrow aspirate and biopsy showing granulocytosis which had proliferated actively, abundant droplet bodies in the cytoplasm, and increased megakaryocytes. $\mathbf{b}$ Anteroposterior radiograph of the upper gastrointestinal tract showing amass in the lower esophagus. c Lateral cephalometric radiograph of the upper gastrointestinal tract showing the lower esophageal mass. $\mathbf{d}$ Chest computed tomography (CT) showing thickening of the esophageal wall with corresponding regions of luminal stenosis. e The resected specimen showing an elevated tumour measuring $6.5 \times 6.0 \times 1 \mathrm{~cm}$ in the lower segment of the esophagus and gastroesophageal junction. f Microscopy, showing a moderately differentiated adenocarcinoma invading the serous layer

was also increased. These results confirmed a diagnosis of LR and excluded chronic myelogenous leukaemia (CML). The patient was referred to our hospital in Jun 2018 for further evaluation and treatment. The patient had an unremarkable family history. On examination, there were no palpable supraclavicular and bilateral cervical lymph nodes, and his body temperature was normal. His vitals were stable with a normal blood pressure, regular heart rate, and normal pulse rate. However, his weight had decreased by $4.5 \mathrm{~kg}$ since he had begun to experienc dysphagia. Laboratory investigations included a routine blood test (total WBC: 29,960/mm3, neutrophils: 89.5\%, lymphocytes: $7.5 \%$, monocytes: $2.3 \%$, and haemoglobin: $138 \mathrm{~g} / \mathrm{l}$ ) and analysis of tumour markers (alpha-fetoprotein: $1.41 \mathrm{ng} / \mathrm{ml}$, squamous cell carcinoma: $0.1 \mathrm{ng} / \mathrm{ml}$, and carcinoembryonic antigen: $1.01 \mathrm{ng} / \mathrm{ml})$. C-reactive protein was elevated (14.36 $\mathrm{mg} / \mathrm{dl}$ ). Yhe uric acid levels and liver and kidney function tests were within normal limits.Hyperleukocytosis, elevated $\mathrm{C}$-reactive protein, and the neurologic features on the day of admission were indicative of an infectious pathology.
Initially, the hyperleukocytosis was considered to be related to an infection. Subsequently, the patient was treated with biapenem ( $0.3 \mathrm{~g}$ twice dailyfor days $1-6)$ and voriconazole (200 mg twice daily for days $1-6$ ). His WBC counts were $25,290 / \mathrm{mm}^{3}$ and $28,730 / \mathrm{mm}^{3}$ on the 4th and 6th days of treatment, respectively. All the blood cultures taken were negative. Persistent leucocytosis after 6 days of antibiotic treatment indicated that this could not be caused solely by infection. We therefore concluded that the LR was most probably caused by esophageal adenocarcinoma. A radiographic examination of the upper gastrointestinal tract (Fig. 1b-c) revealed a mass in the lower esophagus. A chest CT (Fig. 1d) showed thickening of the wall of the esophagus, corresponding regions of luminal stenosis, and several enlarged lymph nodes around the lesser curvature of the esophagus. No primary or metastatic tumours were observed. An abdominal ultrasound was performed, which identified no metastases in the liver. A radical esophagectomy was then performed. The resected specimen showed an elevated tumour measuring $6.5 \times 6.0 \times 1 \mathrm{~cm}$ in the lower 
segment of the esophagus and gastroesophageal junction (Fig. 1e). Microscopically, the tumour was a moderately differentiated adenocarcinoma invading the serous layer (Fig. 1f). A prominent intravascular proliferation of tumour cells was observed, which occasionally formed tumour thrombi. Clearance of lymph nodes during the radical esophagectomy revealed no grossly enlarged nodes. The tumour was diagnosed to be of stage IIB (T3NOM0), according to the American Joint Committee on Cancer guidelines.

Postoperatively, the WBC counts successively decreased to $10,450 / \mathrm{mm}^{3}$ within 1 week and $8670 / \mathrm{mm}^{3}$ at 1 month. The patient received 4 cycles of nedaplatin (200 $\mathrm{mg}$ on day 1 ), fluorouracil (1.0 g on days $1--5)$, and calcium folinate $(0.3 \mathrm{~g}$ on days $1--5)$, and has been free of recurrence for 6 months.

\section{Discussion and conclusion}

A WBC count exceeding $50,000 / \mathrm{mm}^{3}$ associated with extramedullary causes is termed a LR. It results in a severe left shift with the presence of immature myeloid cells in the peripheral blood [1]. Experts now agree that it is not essential the WBC count exceeds $50,000 / \mathrm{mm}^{3}$ to be diagnose LR; counts beyond the normal upper limit $\left(4000-10,000 / \mathrm{mm}^{3}\right)$ are sufficient [1]. Some of these cases have been misdiagnosed as acute leukaemia due to the severe elevation in the WBC count. However, leukaemia commonly presents with anaemia and immature myeloid cells on a peripheral blood smear, and bone marrow biopsies typically show hypercellularity. [2, 9] The evidence is available directly by bone marrow biopsy and by an inverse correlation between tumour response and leucocytosis, as well as an increased PLR [10]. LR typically presents with elevated levels of mature polymorphonuclear cells and immature cells of granulocyte lineage on peripheral smears. Leucocyte alkaline phosphatase is typically normal or elevated in LR. Leucocytosis is a typical response to infections, drugs, chromosomal abnormalities, and paraneoplastic phenomen [11]. LR can also be a paraneoplastic manifestation of several cancers [3]. In solid tumours, LR can be caused by an increased granulocyte colony stimulating factor or other growth factors including granulocyte-macrophage colony-stimulating factor (GM-CSF) and interleukin-6 (IL-6), which is thought to be produced by malignant cells of the cervix, pancreas, hepatocellular carcinoma, and lung cancer [3]. In the normal physiologic state, GM-CSF can stimulate proliferation and differentiation of neutrophil colony-forming cells. GM-CSF can also alter several functions of mature neutrophils [12]. During LR, over-expression of GM-CSF is associated with subsequent leucocytosis [13]. Mari $\mathrm{K}$ et al. reported a patient with esophageal squamous cell carcinoma (ESCC) with remarkable leucocytosis and high G-CSF levels [14]. ESCC is a disease with a poor prognosis, and the prognosis of ESCC with LR is considered even poorer [14]. Shun et al. also reported a case of aggressive G-CSF producing ESCC with increased WBC, and neutrophil counts, and increased C-reactive protein(CRP) [15]. The counts of WBC and neutrophils, and serum CRP levels may be markers of its progression. The neutrophil counts are especially important as a marker of progression [15]. Antoine et al. reported that leucocytosis and neutrophilia were independent prognostic factors of poor overall survival (OS), progression free survival (PFS), and locoregional control (LRC) in patients with locally advanced ESCC undergoing exclusive chemoradiation [16]. Animal experiments have demonstrated that IL-6 can sustain neutrophil/macrophage colonies in vivo, and IL-6 can act on other immune cells, which may induce the production of several CSFs by the bone marrow [17]. Due to the rarity of PLR, studies on this disease have been very limited. HurtadoCordovi J et al. reported that only 77 (10\%) among 758 patients who had solid tumours with hyperleukocytosis in their cohort had PLR [18]. The prognosis of patients with PLR is poor. According to a review, $78 \%$ patients died within 12 weeks of the first detection of hyperleukocytosis; only $10 \%$ of patients were observed to survive more than 1 year after successful treatment of the malignancy. Owing to the poor prognosis of patients with PLR, it is essential to rule out life-threatening causes prior to making a diagnosis of PLR. In terms of early diagnosis and subsequent disease management, staining for GM-CSF early in the course of the disease may provide valuable information. Treatment strategies are limited in patients with PLR, and these are rarely reported in the literature. The effective treatment for leucocytosis is removal of the inciting tumour. Unfortunately, extirpation is not always definitive [19]. In a retrospective, single-institution study, Granger and Kontoyiannis reported that patients with PLR who survived longer than 1 year had received effective antineoplastic therapy, chemotherapy, or surgery [20]. Moreover, it has been reported that $78 \%$ of patients either died or were discharged to hospice within 12 weeks of their initial extreme leucocyte count findings [21]. Here, we report a case of esophageal adenocarcinoma with an abnormal haematological picture dominated by LR. Our patient, underwent radical esophagectomy. Postoperatively, his WBC counts decreased to normal values, and he has been free of recurrence for 6 months.

In conclusion, an accurate and timely diagnosis of LR associated with esophageal adenocarcinoma and appropriate treatment strategies can improve the prognosis of patients with this rare condition.

\footnotetext{
Abbreviations

CML: Chronic myelogenous leukemia; CRP: C-reactive protein; CT: Computed tomography; ESCC: Esophageal squamous cell carcinoma; GMCSF: Granulocyte-macrophage colony-stimulating factor; HCC: Hepatocellular carcinoma; IL-6: Interleukin-6; LR: Leukemoid reaction; LR: Locoregional control; OS: Overall survival; PFS: Progression free survival; PLR: Paraneoplastic Leukemoid reaction; WBC: White blood cell count
} 


\section{Acknowledgements}

Not applicable.

\section{Funding}

Not applicable.

\section{Availability of data and materials}

The datasets during and/or analysed during the current study available from the corresponding author on reasonable request.

\section{Authors' contributions}

GY, HJ and JJ collected the data, designed the report, and drafted the first version. JJ revision the article. $L Z, G G$ and $C L$ critically read the draft. SW, CM and $\mathrm{YH}$ supervised the data collection and drafted subsequent versions of this manuscript. All authors read and approved the final version of the manuscript.

\section{Ethics approval and consent to participate}

The ethics committee of Weihai Municipal Hospital had approved the study.

\section{Consent for publication}

Written informed consent was obtained from the patients for publication of this study. Copies of the written consent are available for review by the Editor-in-Chief of this journal.

\section{Competing interests}

The authors declare that they have no competing interests.

\section{Publisher's Note}

Springer Nature remains neutral with regard to jurisdictional claims in published maps and institutional affiliations.

Received: 23 January 2019 Accepted: 1 April 2019

Published online: 08 April 2019

\section{References}

1. Chakraborty S, Keenportz B, Woodward S, Anderson J, Colan D. Paraneoplastic leukemoid reaction in solid tumors. Am J Clin Oncol. 2015; 38(3):326-30.

2. Sakr R, Renneville A, Saada V, Cotteret S, Martin JE, et al. Next-generation sequencing discriminates myelodysplastic/myeloproliferative neoplasms from paraneoplastic leukemoid reaction in cancer patients with hyperleukocytosis. Leuk Lymphoma. 2018:59(7):1742-5.

3. Jain P, Roop RR, Jha A. Hepatocellular carcinoma manifesting as cavitary lung metastases and leukemoid reaction. J Gastrointestin Liver Dis. 2008; 17(3):355-6.

4. Hughes W, Higley CS. Marked leukocytosis resulting from carcinomatosis. Ann Intern Med. 1952;37:1085-8.

5. Kuş AB, Sahin $P$, Uğuz AH, Tanyeli $A$, Küpeli S. Leukemoid reaction associated with pediatric nasopharyngeal carcinoma: an unusual presentation. Int J Pediatr Otorhinolaryngol. 2014;78(5):885-7.

6. Lee DW, Teoh DC, Chong FL. A case of nasopharyngeal carcinoma with Paraneoplastic Leukemoid reaction: a case report. Med J Malays. 2015;70(2):110-1.

7. Halkes CJ, Dijstelbloem HM, Eelkman Rooda SJ, Kramer MH. Extreme leucocytosis: not always leukaemia. Neth J Med. 2007;65:248-51.

8. $\quad$ Bin LI, Jingchen LU, Dan HE, Wang J, Zhou H, et al. Rapid onset lung squamous cell carcinoma with prominent peritoneal carcinomatosis and an eosinophilic leukemoid reaction, with coexistence of the BRAF V600E and oncogenic KRAS G12A mutations: a case report. Oncol Lett. 2014;8(2):589-93.

9. Hoofien A, Yardenbilavski H, Ashkenazi S, Chodick G, Livni G. Leukemoid reaction in the pediatric population: etiologies, outcome, and implications. Eur J Pediatr. 2018;177(7):1029-1036.

10. Berdel WE, Danhauser-Riedl S, Steinhauser G, Winton EF. Various human hematopoietic growth factors (interleukin-3, GM-CSF, G-CSF) stimulate clonal growth of nonhematopoietic tumor cells. Blood. 1989;73(1):80-3.

11. Hoffman R, Benz EJ, Shattil SJ, Furie B, Cohen HJ. Hematology: basic principles and practice. American Journal of Clinical Pathology. 1995;104(3): 354-355.

12. Shalom G, SionVardy N, Dudnik J, Ariad S. Leukemoid reaction in lung cancer patients. Isr Med Assoc J. 2010;12(4):255-6.
13. Nakamura T, Hatanaka D, Kusakari M, Kashima K, Takizawa Y, et al. Neonatal Leukemoid reaction with fetal inflammatory response syndrome is associated with elevated serum granulocyte Colony stimulating factor and Interleukin-6. Tohoku J Exp Med. 2018;244(2):145-9.

14. Mari K, Yukinori Y, Asami T, Kouichi Y, Susumu A, et al. Radical esophagectomy for a 92-year-old woman with granulocyte colonystimulating factor-producing esophageal squamous cell carcinoma: a case report. World J Surg Oncol. 2016;14(1):264.

15. Shun Y, Kengo K, Shinichiro K, Yasuhiro N, Naoe K, et al. Severe neutrophilic leukocytosis as a progression marker in granulocyte colony-stimulating factor-producing squamous cell carcinoma of the esophagus. Clin Case Rep. 2017;5(5):688-93.

16. Antoine $\mathrm{S}$, Laurence $\mathrm{MZ}$, Eleonor $\mathrm{R}$, Alexandre $\mathrm{E}$, Michel $\mathrm{D}$, et al. Leukocytosis and neutrophilia predict outcome in locally advanced esophageal cancer treated with definitive chemoradiation. Oncotarget. 2017:8(7):11579-11588.

17. Kumar AK, Satyan MT, Holzbeierlein J, Mirza M, Veldhuizen PV. Leukemoid reaction and autocrine growth of bladder cancer induced by paraneoplastic production of granulocyte colony-stimulating factor--a potential neoplastic marker: a case report and review of the literature. J Med Case Rep. 2014; $8(1): 147$.

18. HurtadoCordovi J, Avezbakiyev B, Frieri M, Freedman L, Gebre W. Cutaneous inflammatory malignant fibrous histiocytoma presenting with a leukemoid reaction: a case report and review of the literature. Case Rep Med. 2012 2012:798629.

19. Granger JM, Kontoyiannis DP. Etiology and outcome of extreme leukocytosis in 758 nonhematologic cancer patients: a retrospective, singleinstitution study. Cancer. 2009;115(17):3919-23.

20. Dukes J, Tierney L. Paraneoplastic leukemoid reaction as marker for transitional cell carcinoma recurrence. Urology. 2009;73:928.

21. Ma X, Li G, Cai Z, Sun W, Zhang F. Leukemoid reaction in malignant bone tumor patients - a retrospective, single-institution study. Eur Rev Med Pharmacol Sci. 2012:16(14):1895-9.
Ready to submit your research? Choose BMC and benefit from:

- fast, convenient online submission

- thorough peer review by experienced researchers in your field

- rapid publication on acceptance

- support for research data, including large and complex data types

- gold Open Access which fosters wider collaboration and increased citations

- maximum visibility for your research: over $100 \mathrm{M}$ website views per year

At BMC, research is always in progress.

Learn more biomedcentral.com/submissions 